\title{
The Application of RFID in Warehouse Management
}

\author{
Hui Tan \\ School of Economics \& Management, Nanjing Institute of Industry Technology, Nanjing, Jiangsu Province, China
} (tanh@ niit.edu.cn)

\begin{abstract}
The paper emphasizes how to set up and operate the RFID in the warehouse work of storage, shelves, transfer, tray adjustment, sorting, delivery and inventory process, particularly introduces the information interaction and seamless docking between the RFID background application system and warehouse management system (WMS). Finally summarizes the function of the RFID warehouse management system can realize.
\end{abstract}

Keywords - warehouse management, RFID, WMS, electronic tag

\section{RFID 技术在仓储管理中的应用}

\author{
谈慧 \\ 南京工业职业技术学院经济管理学院, 南京, 江苏, 中国
}

摘 要 首先强调了 RFID 在仓储管理中的管理对象和布局, 重点论述了 RFID 在仓储作业中入库、上架、移库、托盘调整、分 拣、出库、盘点等流程中如何设置和操作, 特别介绍了 RFID 后台应用系统和仓储管理系统 (WMS) 之间的信息交互、无缝对接, 最 后总结了 RFID 仓储管理系统可实现的功能。

关键词 仓储管理, RFID, WMS, 电子标签

\section{1. 引言}

随着现代物流建设的发展, 计算机信息技术、自动化 技术等新兴科技被越来越多地应用到仓储管理当中, 相当 一部分企业的仓库管理已经开始使用仓储管理系统 (Warehouse Management System, WMS) 并采用条码标签 进行自动识别。而 RFID 技术作为条码技术后的新一代的 识别技术, 以快速扫描、无障碍阅读、可重复使用、容量 大、安全性高等特点, 正在深刻地影响着供应链与物流仓 储管理, 借助其快速、实时、准确的信息采集和处理, 可 实现物流仓库标准化和高效化的运营[1]。

\section{RFID 在仓库中管理的对象及任务}

RFID 的应用是在现有仓储管理的到货、验收、入库、 移库、出库等作业环节中进行货品、数量、位置、载体等 信息的实时自动采集, 并通过与 WMS 的信息交互, 在操 作现场提供下一步的操作指示和执行情况校验[2], 从而提 高一线运作的效率和准确性, 实现仓储管理的智能化和自 动化。
2.1 管理对象

企业仓库中 RFID 技术主要用于管理的对象有:

(1) 库存品: 它是仓库管理的根本对象。库存品按存在形 式分为: 托盘、箱体和散装三种形式, 局限于目前 RFID 还不适合管理到每一个细小的单件物品, 因此使用 RFID 管理物品的单位是整箱和整个托盘 (含大件单 品)。

(2) 库位: 仓库中用来摆放库存物品的、在空间上互不重 叠的区域, 一般一个库位可以摆放多个库存品, 也可 以是一个较大的库存品占用多个库位。

(3) 库管设备: 用于仓库管理的设备, 如叉车、手推车等。 在大型的、繁忙的仓库中需要对这些设备进行合理调 度、定时定位, 以提高设备的利用率。

2.2 管理任务

(1) 给每一库位贴电子标签, 该标签称为库位标签。在进 行仓储作业时, 读取该标签编号, 就可判定当前作业 的位置是否正确。 
(2) 在物品入库时, 给每个库存管理物品贴电子标签, 该 标签为货物标签。贴放标签的物品应该是整托盘、整 箱或便于安装标签的大件物品。在进行库房作业时, 读取标签的编号, 确定作业物品是否正确。

(3) 架设无线网络, 覆盖整个仓库作业区, 使所有作业数 据实时传输。在高位叉车上安装固定的无线数据终端, 液压托盘操作人员配手持式无线数据终端。无线数据 终端具有接受作业指令、确认作业位置与作业货物是 否准确等功能[3]。

(4) 使用自动导引车(Automatic Guided Vehicle, AGV)作为 平台, 在上面安装 RFID 识读器、控制设备、无线通 信设备。安装识读器设备的 $\mathrm{AGV}$ 车, 每天在设定时间 内自动对库房进行盘点, 并把盘点结果传输给系统管 理中心。

\section{3 设备部署}

RFID 设备在仓库管理中的部署如图 1 所示。

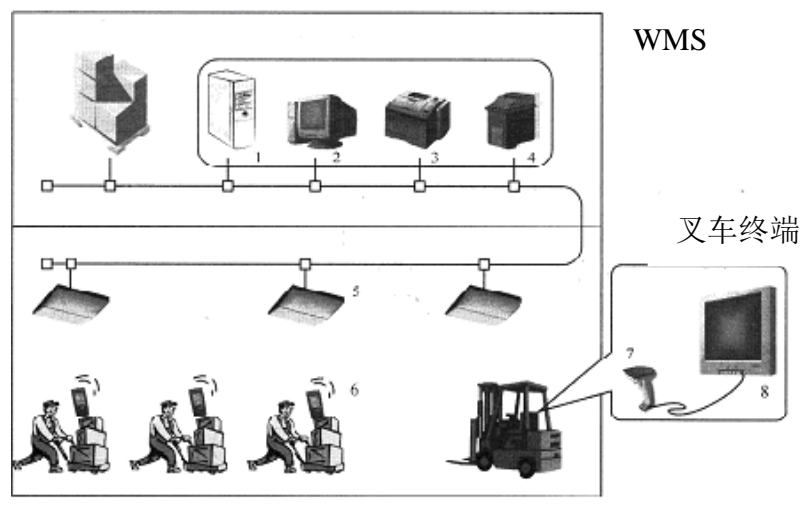

图 1 RFID 设备在仓库管理中的部署

1一库存管理数据库计算机服务器 2 -无线控制, 办公室计算机 3-入库标签打印机 4-激光打印机 5-无线天线 6 -叉车无 线设备终端 7-长距离无线条形码扫描器 8-手持无线终端

\section{RFID 在作业流程中的作用}

本方案将 RFID 后台应用系统和仓储管理系统 (WMS) 无缝结合, 通过 RFID 设备在作业过程中的实时操作达到 数据的快速交换, 实现物流企业出入库管理的安全、高效、 智能化。

\section{1 入库任务指派}

(1) WMS 根据预先设置的规则自动为 “入库单” 分配货位, 产生“入库作业任务单”, 这些单据自动分配给理货组,
并发送信息至理货班长手持 PDA 上, 经确认将信息反 馈至 WMS，此时收货作业启动。

(2) WMS 将 “入库作业任务单” 分解为多组上架指令, 并 按货位的分类（首层、非首层）分别发送至相应的液 压车员工手持 RFID 终端或高位叉车的车载 RFID 读写 终端。

\section{2 入库上架}

(1) WMS 中的地址分配模块通过入库区固定式 RFID 读写 设备为每个托盘分配的货位信息写入托盘电子标签 内。

(2) 托盘车员工操纵托盘车接近托盘时, 车载或手持终端 读取托盘电子标签信息, 显示该托盘的目标货位, 操 作人员将托盘运至货位所在存储区。

(3) 高位叉车司机操纵叉车靠近待上架托盘时, 叉车上的 RFID 终端读取托盘电子标签信息后, 将托盘货物放置 到指定的货位上，并读取货位电子标签信息。

(4) WMS 自动核对托盘与货位关联的正确性, 如相符则反 馈回 WMS, 视作完成上架作业, 如不相符, 则发出声 音警示和错误状态提示, 以便作业人员核对后重新正 确上架。

(5) 上架作业完成后, “入库任务单” 和 “入库单” 完成, 更新 WMS 数据, 并向业务管理系统传送入库数据。

\section{3 托盘拆拼}

（1）拆盘时, 作业人员用手持 RFID 读写器终端读取托盘电 子标签相关信息, 读取移出货物的条码, 并将信息反 馈 WMS，解除原有货物/托盘关联。

(2) 拼盘时，用手持 RFID 读写终端读取移入托盘相关信 息, 读取移入货物的条码, 并将信息反馈 WMS, 重新 建立新的货物/托盘关联, 更新 WMS 中的库存和货位 信息。

\section{4 货位调整}

(1) 车载或手持 RFID 读写终端分别读取移出托盘和移出 货位的电子标签信息, 解除该货位与托盘的关联关系。

(2) 叉车司机或液压车员工把移出托盘搬运至新货位。

(3) 车载或手持 RFID 终端读取移入货位电子标签相关信 息, 重新建立移入托盘与移入货位的关联关系, 并将 信息反馈 WMS, WMS 实时更新库存信息, 并将更新 后的库存数据发送到业务管理系统。 
3.5 移库调度

(1) 货主在联网的业务系统中录入 “移库申请单”, 并发送 至物流企业WMS。

(2) WMS 对 “移库申请单” 进行处理, 产生以承运车辆为 单位的 “干线物流任务单”, 以此为依据申领准运证, 然后以货主为单位生成 “移库出库单”, 并反馈回管理 系统。

3.6 出库任务指派

(1) WMS 将已分配好下架货位的 “出库作业任务单”, 根 据一定逻辑关系自动分配至理货组、托盘车组和叉车 司机，形成作业策略。

(2) 将分配方案发送至理货组班长手持 PDA 上, 理货班长 确认或修改分配方案, 并将信息发送回 WMS。WMS 将出库作业任务单发至指定理货人员手持 PDA 上, 理 货人员进行签到确认, 将相关信息反馈至 WMS, 并安 排做好装车准备。

(3) WMS 按货位的分类将出库作业任务单分别发送至相 应的托盘车员工的手持终端或高位叉车的车载 RFID 读写终端, 相应的作业人员按指令进行实物的下架操 作。

\section{7 出库拣货下架}

(1) 当有货物出库时, 叉车司机根据指令靠近指定的货位, 车载 RFID 读写终端（或手持 RFID 读写终端）读取货 位电子标签的相关信息和货位上托盘电子标签的相关 信息, 即时进行下架指令、货位与托盘三者的匹配性 校验, 确认无误后取下托盘, 完成托盘下架动作, 并 将相关信息反馈至 WMS 后台。

(2) 出库货物数量不是满托盘时, 先按上述方法取下托盘, 提取需要出库数量的货物, 转移到另一空托盘上, 并 按拆盘规则操作, 剩余货物的托盘由 WMS 安排合适的 货位存放并更新相关信息。

\section{8 移库出库理货}

(1) 液压车员工靠近已下架托盘时, 用手持 RFID 手持终端 读取托盘电子标签信息, 核对托盘上货物的货主、品 种、规格、数量, 确认无误后, 按照 WMS 指定的码头 号码, 用托盘车将货物运至指定码头前的出库暂存区 等待装车。

(2) 理货人员对在出库暂存区的货物进行复核, 用手持 PDA 采集每个托盘上任意一货物的条码, 在 WMS 上 获得该货物所有托盘上所有货物的数据, 累计后与 “出
库任务单” 自动进行核对, 当出现差异时, WMS 在固 定终端、移动终端发出警报。

\section{9 分拣出库交接}

（1）智能调度系统把零售客户订单信息（包括品种、规格、 数量、线路、顺序等) 导入 WMS, 生成分拣出库单。

(2) WMS 从分拣系统中导入分拣进程状态, 按循环补货的 策略和出库货位分配策略, 将分拣出库任务单拆分生 成出库作业任务单, 并向各作业人员下达拣货下架指 令。

（3）托盘下架后, 作业人员靠近目标托盘, 用手持 RFID 终 端读取托盘电子标签信息 (货主、品种、规格、数量、 出库类型等), 进行分拣出库指令与托盘的校验, 校验 无误后根据指令将整托盘货物经过规定通道运送至分 拣区指定位置。

(4) 整托盘货物经过规定通道入口时, 设在入口处的 RFID 读写终端自动读取托盘电子标签中货物条码数据包, 通过解密解析得到对应货物条码信息, 将托盘电子标 签中的货物条码信息上传给 WMS, 由 WMS 系统及时 反馈给扫码系统, 完成分拣前的再次确认, 同时数据 回传 WMS, 在 WMS 中作分拣出库完成的处理, 进入 分拣配货流程。

3.10 盘点

盘点前, 调度人员选择所需的盘点策略, 生成相应的 盘点指令。实盘时, 盘点人员用手持 RFID 终端读取货位 电子标签信息, 回传至 WMS, 从 WMS 中获取该货位上的 货物全部库存信息, 盘点人员通过手持 RFID 读写终端上 显示的信息, 与现场实物进行核对, 采集完毕后把数据上 传到 WMS 中[4]。盘点完成后由 WMS 生产盘点表和盈亏 表。

\section{RFID 系统功能模块设计}

基于 RFID 的仓储管理系统主要功能模块设计如下:

\section{1 基础数据管理}

(1) 对系统中数据库的集中管理与维护。可以对计量单位, 商品品种, 日历, 系统参数, 叉车, 人员信息等进行 维护。

（2）供应商注册, 供应商品名和买价设置; 客户注册, 客 户品名和卖价设置。

（3）可查询买价卖价对照表、物品一览表、库物品库龄报 表、商品进出一览表、库存及运输月报表查询等。 
4.2 订单管理

(1) 入库订单管理。实现货物入库操作, 可完成人库单据 的录入、修改、删除, 入库货位自动分配、手动分配。 已分配货位单据按指令发送等作业。

(2) 出库订单管理。和入库订单管理类似, 实现货物出库 操作。

(3) 查询。实现客户订单查询, 货位调整单查询、入库单 查询、出库单查询、货运状态查询, 库存查询等, 并 可打印生成各种管理报表。

4.3 库位管理

（1）电子货位图管理。主要用来查询和显示仓库货位存储 状况及进行可视化的货位调整操作, 包括库区适时库 存货位信息显示、货物(托盘)的库存地址查询、调整等 操作。

(2) 入库标签管理。发行入库标签, 进行实时库存管理, 通过无线通信网络发布仓库管理作业指令。

(3) 货物组托管理。仓储系统支持非整托盘入 / 出库, 所 以入 / 出库作业需要组盘功能模块以实现入库组盘、 出库拆盘的操作。组盘功能模块主要实现读写托盘 RFID 标签信息、托盘货物增 / 减、组盘撤销等功能。

\section{4 业务执行管理}

(1) 完成入库、出库、移库、调拨、盘库等作业的具体操 作，可根据任务单查询每个环节的执行情况。

（2）货物盘点。支持两种盘点方式: 普通盘点和循环盘点。 普通盘点时必须将所有作业暂停; 而后者可以在大部 分作业进行时盘点, 但需要实时设备(例如 $\mathrm{RF}$ ) 的支持。

\section{RFID 系统可实现的功能}

\section{1 快捷的出入库管理}

在库房入口处安装固定的 RFID 读取设备或通过手持 设备自动对入库的货物进行识别, 如果每个包装上均安装 有电子标签, 可以识别到单品, 同时由于 RFID 的多读性, 可以同时批量识别多个标签[5], 出入库速度大大提高。

\section{2 可视化库存管理}

在系统中点击每个货位, 可实时查询货架库存, 并显 示相应库位图。每件货品上都有内含电子芯片的标签, 可 及时了解库存信息, 便于控制合理库存。

\section{3 快速盘点和准确查找}

在货架或者专属货位上贴有电子标签, 对货位进行精 确定位, 通过货架标签和托盘标签可以实现快速盘点, 同 时由于货架标签和货架上存放物品的对应关系可以快速准 确的找到货物具体位置。

\section{4 叉车作业智能化}

在叉车上安装专用的 RFID 读写器和显示器, 通过系 统向司机发送指令, 叉车司机只需要按照系统提供的命令 进行作业, 快捷、准确, 不易出错。

\section{5 智能调度物流车辆}

通过物流运输 GPS 监控服务管理系统, 合理调用物流 运输车辆, 随时了解车辆的在途行使状况及周边环境。

\section{6. 结束语}

基于 RFID 技术的仓储管理围绕电子标签和物联网这 两个核心, 通过电子标签实现产品的自动识别, 利用物联 网获取产品原始信息并自动生成入库和出库清单, 从而为 自动化的仓储管理提供了一种行之有效的手段。本文分析 的 RFID 技术在物流企业仓储管理中的解决方案, 已在南 京陆通物流有限公司实施运行, RFID 系统上线后, 数据记 录错误率减少了 $80 \%$; 货物的吞吐量增加了 $35 \%$, 物流作 业水平和效率大大提升, 实现了仓储管理能力和仓储服务 水平的倍增。

\section{参考文献(References)}

[1] Tan Hui, "Application of RFID System in Manufacturing Production Control", the manufacturing industry informatization of China, pp.13-16, April 2010.

[2] Tan Hui, "Analysis and Design of Third Party Logistics Management Information System", the management informatization of China, pp.62-65, April 2008.

[3] Zhang Feizou. Yang Dongkai, Internet of Tings: Applications and Solutions, Beijing: Publishing House of Electronics Industry, 2012.

[4] Hu Fengbin, "Web-based Third-party Logistics System Design", Computer Information and Technology, pp.109-110, May 2006.

[5] You Zhanqing, etc., Theory and Application of Radio Frequency Identification Technology (RFID), Beijing: Publishing House of Electronics Industry, 2004. 